\title{
DYNAMIC ANALYSIS OF A ROD VIBRO-IMPACT SYSTEM WITH INTERMEDIATE SUPPORTS
}

\author{
Volodymyr GURSKY*, Igor KUZIO \\ *Department of Mechanics and Automation Engineering, Institute of Engineering Mechanics and Transport, \\ Lviv Polytechnic National University, 12 Bandera Street, 79013 Lviv, Ukraine \\ vol.gursky@gmail.com, ikuzio@polynet.lviv.ua
}

received 28 July 2016, revised 8 June 2018, accepted 12 June 2018

\begin{abstract}
The two-mass resonant vibro-impact module is presented as the rod system with cylindrical intermediate supports. The corresponding design diagram is constructed. Based on the finite element method, the frequency of free oscillations is defined for the corresponding location of the intermediate supports. A stress-strain state of the elastic element is considered. The stiffness of the intermediate supports is defined by solving the contact problem between the cylindrical rod supports and the flat spring. The dynamics of the vibro-impact rod system with multiple natural frequencies is analyzed taking into account the contact stiffness of the intermediate supports. The determination of contact and equivalent stresses occurring during the operation of the vibro-impact rod system is performed.
\end{abstract}

Key words: Rod System, Electromagnetic Drive, Vibro-impact, Resonance, Finite Element Method (FEM)

\section{INTRODUCTION}

Vibromachines are widely used in industries with large energy consumption, especially in metallurgy, civil and mechanical engineering (Bednarski and Michalczyk, 2017; Nadutyi et al., 2013; Filimonikhin and Yatsun, 2017). This emphasizes their actuality. A large amount of vibromachines are of the vibro-impact type and fall under the category of nonlinear systems (Babitsky, 2013). They can operate both in non-resonant and in resonant mode (Luo et al., 2007; Sokolov et al., 2007). Resonant systems are energy efficient ones, so they have better prospects, despite the peculiarities of their operational parameters control (Despotović et al., 2014). Their structural basis is formed by nonlinear stiffness characteristics chiefly by piecewise-linear asymmetric characteristics (Krot, 2010; Yoon and Kim, 2015). They are the most usable ones in terms of implementation. Several elastic elements working in series are applied to implement an appropriate stiffness characteristic. One of them is a working element and the other one is a nonmetallic (rubber) movement limiter. The system is to be setup in order to ensure the resonance operation mode by changing the value of the stiffness coefficient of the metallic elastic element.

\section{DESCRIPTION OF THE STRUCTURE OF THE VIBRO-IMPACT SYSTEM}

Fig. 1 shows a general view of a vibro-impact resonance module with an electromagnetic drive. It consist of two oscillatory masses (working mass 1 and reactive mass 2 ), which have the values of $m_{1}$ and $m_{2}$ and are joined by one flat spring 3 . The oscillatory mass 1 is designed as a frame (body); the brackets 4 are fixed in their horizontal grooves. The metallic or non-metallic cylindrical rods 5 , on which the flat spring 3 is lent, are assembled in vertical grooves of the brackets 4 . The use of such movement limiters allow to implement various asymmetric characteristics of stiffness using one flat spring 4. Two alternating current electromagnets presented in a form of cores with coils 6 and armatures 7 , which are placed on corresponding oscillatory masses, are used for system disturbance.

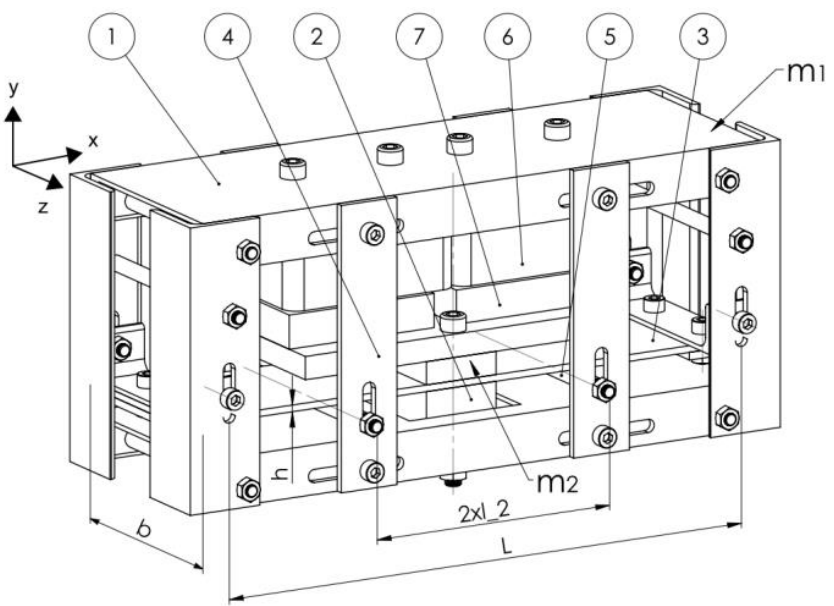

Fig. 1. General view of the two-mass resonance vibratory module: 1 - working mass, 2 - reactive mass, 3 - flat spring, 4 - brackets, 5 - intermediate supports (cylindrical rods),

6 - coils of electromagnets, 7 - armatures

The approach proposed for implementation of an asymmetric stiffness characteristic (Fig. 2) consists in the use of the limiter in a form of absolutely rigid supports contacting with the resonance elastic element. Herewith, the rational implementation of an asymmetric characteristic of a single stiffness element is possible 
under the condition of coordination of the structural parameters with the frequency parameters of a vibratory machine, as well as with the tractive characteristic of a drive.

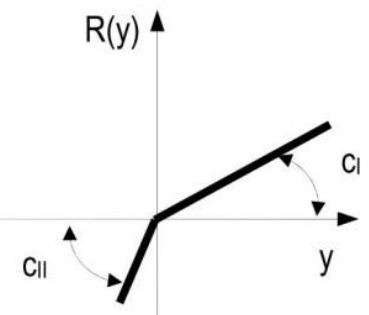

Fig. 2. Piecewise-linear asymmetric stiffness characteristic of a flat spring with intermediate supports

Investigations on the subject of dynamics and synthesis of the vibro-impact systems based on piecewise-linear asymmetric stiffness characteristics are presented in many research papers. Despite the fact that much attention is paid to the problems of dynamic analysis, in particular to the stability analysis and the study of nonlinear effects in a form of bifurcations and subresonances (Simon et al., 2014; Belovodskiy et al., 2015), it is essential to coordinate the main frequency and force characteristics of a particular design diagram with the appropriate inertial and geometrical parameters.

\section{FEM MODEL AND FREQUENCY ANALYSIS}

Considering the vibratory machines as the dynamic models, while carrying out the calculations, they can be presented as the rod systems (elements) with distributed or lumped parameters.

Fig. 3 shows an operational diagram of a resonance vibroimpact module implemented in a form of a rod system. The upward movement is implemented in diagram I without supports; the downward movement is represented in diagram II with rigid intermediate supports. The structural non-linearity in a form of the gapless asymmetrical stiffness characteristic (Fig. 2) is implemented with a help of a flat spring of bending stiffness $E J$ using rigid or relatively compliant movement limiters fixed on appropriate parts of an elastic element. Herewith, the real two-mass system can be transformed to the single mass system with the corresponding mass reduction to an inertial coefficient $m=\frac{m_{1} m_{2}}{\left(m_{1}+m_{2}\right)}$. Thus, the procedure of dynamic analysis is to be substantially simplified, in particular by the generalized coordinate $y(t)$.

Vibro-impact processes are attended by contact phenomena both in the mediums being treated and in the mechanical systems, in particular at the moment of switching the stiffness characteristic from the parameter $c_{I}$ to the parameter $c_{I I}$. Thus, it is important to take into account these effects while carrying out the complex analysis of a vibratory system for representation of the dynamic problem integrity and for studying the efficiency of the vibroimpact mode implementation.

The problems of frequency analysis of the elastic rod systems can be solved directly by analytical methods, as well as by approximate methods (Clough and Penzien, 1995) performing the finite and boundary elements analysis, or using the methods of Relay, Dunkerley, etc. The finite element method (FEM) has become very popular (Hutton, 2004) and it is the basis of all applications of computer analysis. Under appropriate conditions, the original problem can be reduced to the final formulas applicable for performing the engineering calculations and the system designing.

a)

b)

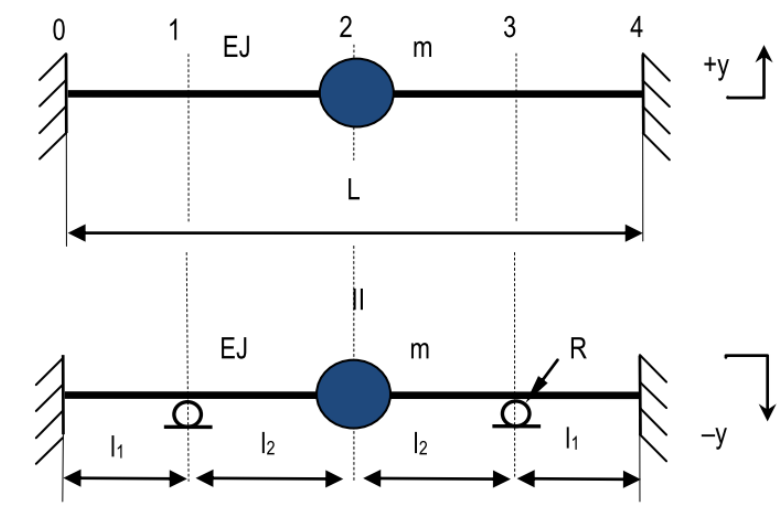

Fig. 3. Operational diagrams of the rod system implementing an asymmetric stiffness characteristic without (a) and with (b) the intermediate supports

The formula for determination of the natural frequency of bending oscillations of the rod system discrete model with the reduced mass $m$ is presented as (Hutton, 2004):

$\omega_{0 I}=\sqrt{192 E J / m L^{3}}$.

To ensure the prescribed natural frequency of free oscillations while performing the design calculation of bending stiffness $E J$, the following formula is used:

$E J=\frac{m L^{3} \omega_{0 I}^{2}}{192}$.

To construct the stiffness matrix of the presented rod system with a help of FEM, it is used the basic element in a form of the rod with four degrees of freedom: $y_{i}, y_{i+1}$ and $\varphi_{i}, \varphi_{i+1}$ are the vertical displacements and the angles of rotation of the utmost nodal points of the rod. The matrix of nodal reactions of the shearing forces and the bending moments is as follows (Hutton, 2004; Clough and Penzien, 1995):

$\left[\overline{\mathbf{Z}}_{i, i+1}\right]=\left\{\begin{array}{c}R_{i} \\ M_{i} \\ R_{i+1} \\ M_{i+1}\end{array}\right\}=$

$E J\left[\begin{array}{cccc}12 / l_{i}{ }^{3} & -6 / l_{i}{ }^{2} & -12 / l_{i}{ }^{3} & -6 / l_{i}{ }^{2} \\ -6 / l_{i}{ }^{2} & 4 / l_{i} & 6 / l_{i}{ }^{2} & 2 / l_{i} \\ -12 / l_{i}{ }^{3} & 6 / l_{i}{ }^{2} & 12 / l_{i}{ }^{3} & 6 / l_{i}{ }^{2} \\ -6 / l_{i}{ }^{2} & 2 / l_{i} & 6 / l_{i}{ }^{2} & 4 / l_{i}\end{array}\right]$.

The matrix of reactions is used for constructing the diagrams of the stress-strain state of the rod system.

The finite element diagram for free bending oscillations analysis of the rod system presented in Fig. 3, b takes into account the corresponding boundary conditions:

$\left(\begin{array}{c}y_{0}=0 \\ \varphi_{0}=0\end{array}\right),\left(\begin{array}{c}y_{1}=0 \\ \varphi_{1}=\operatorname{var}\end{array}\right),\left(\begin{array}{l}y_{2}=\text { var } \\ \varphi_{2}=\text { var }\end{array}\right)$,
$\left(\begin{array}{c}y_{3}=0 \\ \varphi_{3}=\operatorname{var}\end{array}\right),\left(\begin{array}{l}y_{4}=0 \\ \varphi_{4}=0\end{array}\right)$. 
The stiffness matrix of $10 \times 10$ order of the separate finite elements-rods is constructed on the basis of the on generalized matrix (3) of the single rod in the absolute coordinate system taking into consideration the boundary conditions presented in (4).

$\mathbf{C}_{01}=E J\left[\begin{array}{cccc}1 & 0 & 0 & 0 \\ 0 & 1 & 0 & 0 \\ 0 & 0 & 1 & 0 \\ 0 & 0 & 0 & 4 / l_{1}\end{array}\right]$

$\mathbf{C}_{12}=E J\left[\begin{array}{cccc}1 & 0 & 0 & 0 \\ 0 & 4 / l_{2} & -6 / l_{2}{ }^{2} & 2 / l_{2} \\ 0 & -6 / l_{2}{ }^{2} & 12 / l_{2}{ }^{3} & -6 / l_{2}{ }^{2} \\ 0 & 2 / l_{2} & -6 / l_{2}{ }^{2} & 4 / l_{2}\end{array}\right]$,

$\mathbf{C}_{23}=E J\left[\begin{array}{cccc}12 / l_{2}{ }^{3} & 6 / l_{2}{ }^{2} & 0 & 6 / l_{2}{ }^{2} \\ 6 / l_{2}{ }^{2} & 4 / l_{2} & 0 & 2 / l_{2} \\ 0 & 0 & 1 & 0 \\ 6 / l_{2}{ }^{2} & 2 / l_{2} & 0 & 4 / l_{2}\end{array}\right]$

$\mathbf{C}_{34}=E J\left[\begin{array}{cccc}1 & 0 & 0 & 0 \\ 0 & 4 / l_{1} & 0 & 0 \\ 0 & 0 & 1 & 0 \\ 0 & 0 & 0 & 1\end{array}\right]$

The stiffness matrix of the rod system with 10 degrees of freedom consisting of four rod elements (5) can be constructed by adding the separate stiffness matrixes in the absolute coordinate system, where the coefficients are to be added taking into account the corresponding generalized coordinates:

$\mathrm{C}_{04}=$

$E J\left[\begin{array}{cccccccccc}1 & 0 & 0 & 0 & 0 & 0 & 0 & 0 & 0 & 0 \\ 0 & 1 & 0 & 0 & 0 & 0 & 0 & 0 & 0 & 0 \\ 0 & 0 & 2 & 0 & 0 & 0 & 0 & 0 & 0 & 0 \\ 0 & 0 & 0 & \frac{4}{l_{1}}+\frac{4}{l_{2}} & \frac{-6}{l_{2}{ }^{2}} & \frac{2}{l_{2}} & 0 & 0 & 0 & 0 \\ 0 & 0 & 0 & \frac{-6}{l_{2}{ }^{2}} & \frac{24}{l_{2}{ }^{3}} & 0 & 0 & \frac{6}{l_{2}{ }^{2}} & 0 & 0 \\ 0 & 0 & 0 & \frac{2}{l_{2}} & 0 & \frac{8}{l_{2}} & 0 & \frac{2}{l_{2}} & 0 & 0 \\ 0 & 0 & 0 & 0 & 0 & 0 & 2 & 0 & 0 & 0 \\ 0 & 0 & 0 & 0 & \frac{6}{l_{2}{ }^{2}} & \frac{2}{l_{2}} & 0 & \frac{4}{l_{1}}+\frac{4}{l_{2}} & 0 & 0 \\ 0 & 0 & 0 & 0 & 0 & 0 & 0 & 0 & 1 & 0 \\ 0 & 0 & 0 & 0 & 0 & 0 & 0 & 0 & 0 & 1\end{array}\right]$

The free oscillations equation of the rod system is written in the matrix form:

$\mathbf{M} \times \ddot{\mathbf{X}}+\mathbf{C}_{04} \times \mathbf{X}=0$

where $\mathbf{X}=\operatorname{column}\left(y_{0}, \varphi_{0}, \ldots, y_{4}, \varphi_{4}\right)$ is the column vector of the nodal finite elements displacements; $\mathbf{M}=\operatorname{diag}(0,0,0,0, m, 0,0,0,0,0)$ is the diagonal matrix of the inertial parameters of the localized masses; $\mathbf{C}_{\mathbf{0 4}}$ is the system stiffness matrix

The frequency equation of the rod system formed on the basis of the determinant

$\operatorname{det}\left(-\mathbf{M} \times \Omega^{2}+\mathbf{C}_{\mathbf{0 4}}\right)=0$,

and allows to derivate the formula for calculation of the natural frequency of free bending oscillations:

$\omega_{0 I I}=\sqrt{6 E J\left(l_{1}+4 l_{2}\right) / m\left(l_{1}+l_{2}\right) l_{2}{ }^{3}}$.

According to the equations (1) and (9), the stiffness coefficients of the rod systems are as follows:

$c_{I}=192 E J / L^{3}, c_{I I}=6 E J\left(l_{1}+4 l_{2}\right) /\left(l_{1}+l_{2}\right) l_{2}{ }^{3}$,

Taking into account that $l_{2}=\frac{L}{2}-l_{1}$, the ratio of the stiffness coefficients can be presented as:

$n_{c}=c_{I I} / c_{I}=L^{2}\left(L-1.5 l_{1}\right) /\left(L-2 l_{1}\right)^{3}$,

The ratio of the natural frequencies of free oscillations is as follows:

$n_{\omega}=\omega_{0 I I} / \omega_{0 I}=\sqrt{n_{c}}$.

For example, introducing the multiplicity condition of the bending oscillations of the considered rod systems as

$n_{\omega}=2$,

the formulas of determination of the intermediate supports location are derived:

$l_{1}=0.275 \mathrm{~L}, l_{2}=0.225 \mathrm{~L}$,

considering that $l_{1}+l_{2}=0.5 \mathrm{~L}$.

Considering the stiffness of the intermediate supports. In fact, the intermediate supports cannot be absolutely rigid (Fig. 4). Their compliance can be influenced by the design of the corresponding limiters made of the non-metallic materials.

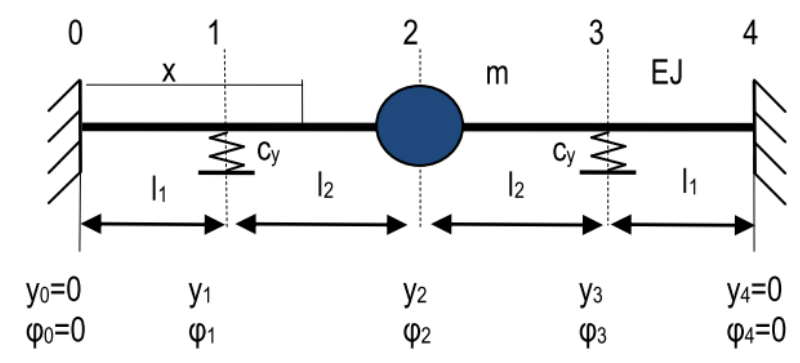

Fig. 4. Computational finite-element diagram and boundary conditions of the rod system with elastic intermediate supports

To take into account the stiffness coefficient $c_{y}$ of the intermediate supports in a vertical direction, the resultant stiffness matrix $\mathbf{C}_{\mathbf{0 4}}$ of the rod system is constructed. It takes into account the corresponding boundary conditions:

$\left(\begin{array}{l}y_{0}=0 \\ \varphi_{0}=0\end{array}\right),\left(\begin{array}{l}y_{1}=\operatorname{var} \\ \varphi_{1}=\operatorname{var}\end{array}\right),\left(\begin{array}{l}y_{2}=\operatorname{var} \\ \varphi_{2}=\operatorname{var}\end{array}\right)$,
$\left(\begin{array}{l}y_{3}=\operatorname{var} \\ \varphi_{3}=\operatorname{var}\end{array}\right),\left(\begin{array}{l}y_{4}=0 \\ \varphi_{4}=0\end{array}\right)$,

and is presented as: 


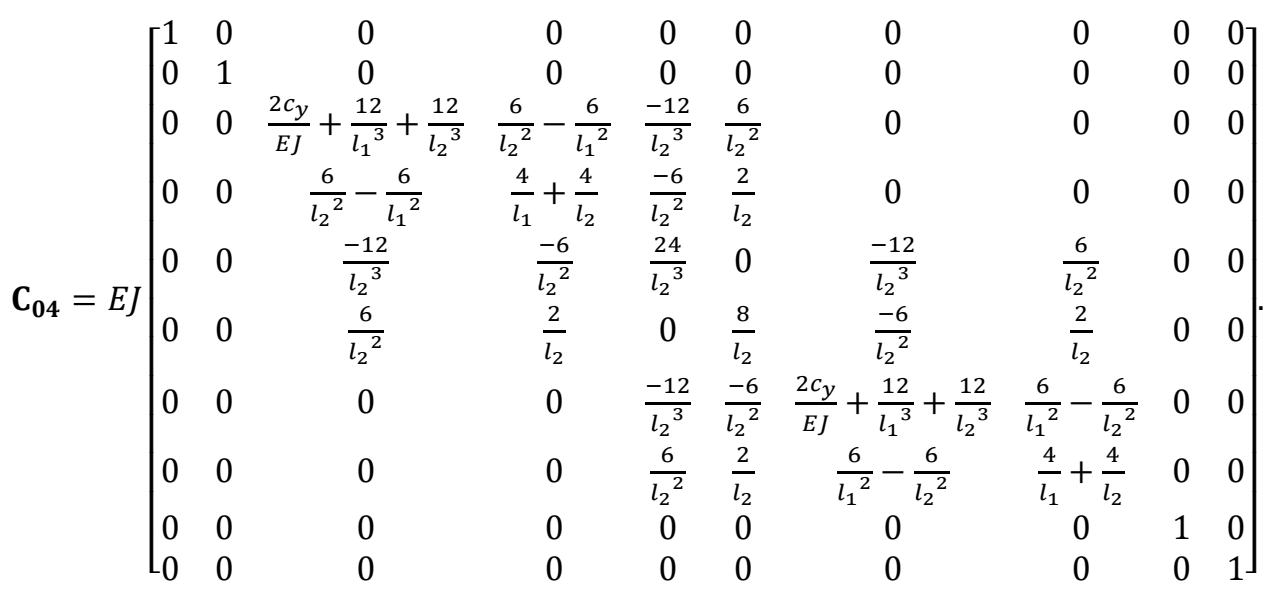

The formula for calculating the natural frequency of free oscillation of the system derived on the basis of the corresponding equation taking into account the stiffness matrix $\mathbf{C}_{\mathbf{0 4}}$ mentioned above:

$\Omega=8 \sqrt{\frac{\left.\begin{array}{c}3 E J \operatorname{Lm}\left(2 c_{y} L l_{1}{ }^{3}-3 c_{y} L^{4}+3 E J L\right) \times \\ 2 c_{y} L^{3} l_{1}{ }^{3}+3 E J L^{3}-12 c_{y} L^{2} l_{1}{ }^{4}+ \\ +24 c_{y} L l_{1}{ }^{5}-16 c_{y} l_{1}{ }^{6}\end{array}\right)}{m\left(\begin{array}{c}2 c_{y} L^{4} l_{1}{ }^{3}+3 E J L^{4}-12 c_{y} L^{3} l_{1}{ }^{4}+ \\ +24 c_{y} L^{2} l_{1}{ }^{5}-16 c_{y} L l_{1}{ }^{6}\end{array}\right)}}$.

The natural frequency of the system $\Omega$ is within $\omega_{0 I} \leq \Omega \leq$ $\omega_{0 I I}$ (Fig. 5) and approaches asymptotically to the value $\omega_{0 I I}$ with considerable increase of the stiffness coefficient $c_{y}$ :

$\lim _{c_{y \rightarrow \infty}} \Omega=\omega_{0 I I}$,

Considering the real support stiffness $c_{y}$ in the case of ensuring the appropriate value of the natural frequency requires adjustment of their location using the appropriate formula (16).

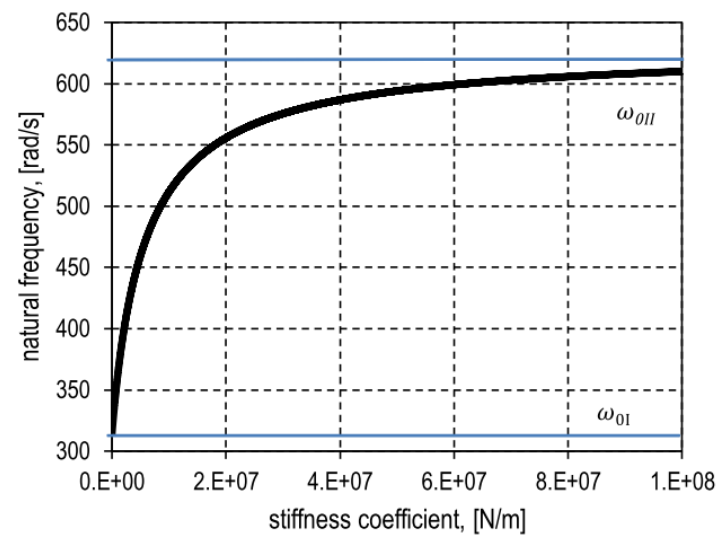

Fig. 5. Influence of the stiffness coefficient of the intermediate supports on the natural frequencies of oscillations of the rod system

The model being analyzed numerically has the following parameters: $\omega=314 \mathrm{rad} / \mathrm{s}, \quad m=20 \mathrm{~kg}, L=0.5 \mathrm{~m}, \quad E=$ $2.05 \times 10^{5} \mathrm{MPa}, b=0.080 \mathrm{~m}, R=0.006 \mathrm{~m}$.

On the basis of the parameters presented above we can calculate the following values: $E J=1.284 \times 10^{5} \mathrm{Nm}^{2}, l_{1}=$ $0.113 \mathrm{~m}, l_{2}=0.137 \mathrm{~m}, h=9.79 \times 10^{-3} \mathrm{~m}, c_{I}=1.972 \times$
$10^{6} \mathrm{~N} / \mathrm{m}, \quad c_{I I}=7.888 \times 10^{6} \mathrm{~N} / \mathrm{m}, \quad \omega_{0 I}=314 \mathrm{rad} / \mathrm{s}$, $\omega_{0 I I}=628 \mathrm{rad} / \mathrm{s}$.

\section{FORCE ANALYSIS AND STIFFNESS CHECK-UP}

The vector of the nodal displacements for statically loaded rod system of mode II can be defined by equation:

$\mathbf{Y}=\mathbf{C}_{\mathbf{0 4}}{ }^{-1} \times \mathbf{P}$,

where $\mathbf{P}$ is the vector of external loads reduced to nodes.

The nodal displacements vectors of each finite element-rod are constructed using the matrix equation (18) in the form of state vectors:

$\mathbf{Y}_{\mathbf{0 1}}=\left[\begin{array}{l}y_{0} \\ \varphi_{0} \\ y_{1} \\ \varphi_{1}\end{array}\right], \mathbf{Y}_{\mathbf{1 2}}=\left[\begin{array}{l}y_{1} \\ \varphi_{1} \\ y_{2} \\ \varphi_{2}\end{array}\right]$,

$\mathbf{Y}_{\mathbf{2 3}}=\left[\begin{array}{l}y_{2} \\ \varphi_{2} \\ y_{3} \\ \varphi_{3}\end{array}\right], \mathbf{Y}_{\mathbf{3 4}}=\left[\begin{array}{l}y_{3} \\ \varphi_{3} \\ y_{4} \\ \varphi_{4}\end{array}\right]$.

The vectors of the nodal reactions of each finite element are determined using the matrix equation (3). Plotting a deflection curve of each finite element-rod is performed based on the known shape functions for unit nodal displacement (Shigley, 2011) defined by (20).

$\mathrm{y}_{01}(x)=\left[\begin{array}{c}\frac{\left(2 x^{3}-3 l_{1} x^{2}+l_{1}{ }^{3}\right)}{l_{1}{ }^{3}} \\ \frac{\left(x^{3}-2 l_{1} x^{2}+l_{1}{ }^{2} x\right)}{l_{1}{ }^{2}} \\ \frac{\left(-2 x^{3}+3 l_{1} x^{2}\right)}{l_{1}{ }^{3}} \\ \frac{\left(x^{3}-l_{1} x^{2}\right)}{l_{1}{ }^{2}}\end{array}\right] \times \mathbf{Y}_{01}$,

$\mathrm{y}_{12}(x)=\left[\begin{array}{c}\frac{\left(2\left(x-l_{1}\right)^{3}-3 l_{2}\left(x-l_{1}\right)^{2}+l_{2}{ }^{3}\right)}{l_{2}{ }^{3}} \\ \frac{\left(\left(x-l_{1}\right)^{3}-2 l_{2}\left(x-l_{1}\right)^{2}+l_{2}{ }^{2}\left(x-l_{1}\right)\right)}{l_{2}{ }^{2}} \\ \frac{\left(-2\left(x-l_{1}\right)^{3}+3 l_{2}\left(x-l_{1}\right)^{2}\right)}{l_{2}{ }^{3}} \\ \frac{\left(\left(x-l_{1}\right)^{3}-l_{2}\left(x-l_{1}\right)^{2}\right)}{l_{2}{ }^{2}}\end{array}\right] \times \mathbf{Y}_{12}$, 


$$
\begin{aligned}
& \mathrm{y}_{23}(x)=\left[\begin{array}{c}
\frac{\left(2\left(x-l_{1}-l_{2}\right)^{3}-3 l_{2}\left(x-l_{1}-l_{2}\right)^{2}+l_{2}{ }^{3}\right)}{l_{2}{ }^{3}} \\
\left.\frac{\left(x-l_{1}-l_{2}\right)^{3}-2 l_{2}\left(x-l_{1}-l_{2}\right)^{2}+}{+l_{2}{ }^{2}\left(x-l_{1}-l_{2}\right)}\right)^{2} \\
\frac{\left(-2\left(x-l_{1}-l_{2}\right)^{3}+3 l_{2}\left(x-l_{1}-l_{2}\right)^{2}\right)}{l_{2}{ }^{3}} \\
\frac{\left(\left(x-l_{1}-l_{2}\right)^{3}-l_{2}\left(x-l_{1}-l_{2}\right)^{2}\right)}{l_{2}{ }^{2}}
\end{array}\right] \times \mathbf{Y}_{23}, \\
& \mathrm{y}_{34}(x)=\left[\begin{array}{c}
\frac{\left(\begin{array}{c}
2\left(x-l_{1}-2 l_{2}\right)^{3}-3 l_{1}\left(x-l_{1}-2 l_{2}\right)^{2}+ \\
+l_{1}{ }^{3}
\end{array}\right)}{l_{1}{ }^{3}} \\
\frac{\left(\begin{array}{c}
\left(x-l_{1}-2 l_{2}\right)^{3}-2 l_{1}\left(x-l_{1}-2 l_{2}\right)^{2}+ \\
+l_{1}^{2}\left(x-l_{1}-2 l_{2}\right)
\end{array}\right)}{l_{1}^{2}} \\
\frac{\left(-2\left(x-l_{1}-2 l_{2}\right)^{3}+3 l_{1}\left(x-l_{1}-2 l_{2}\right)^{2}\right)}{l_{1}{ }^{3}} \\
\frac{\left(\left(x-l_{1}-2 l_{2}\right)^{3}-l_{1}\left(x-l_{1}-2 l_{2}\right)^{2}\right)}{l_{1}^{2}}
\end{array}\right] \times \mathbf{Y}_{34} \text {. }
\end{aligned}
$$

The total displacements and the rod deflection curve of the system II are determined using the following dependences:

$$
y(x)=\left\{\begin{array}{c}
y_{01}(x), \quad 0 \leq x \leq l_{1}, \\
y_{12}(x), \quad l_{1}<x \leq l_{1}+l_{2}, \\
y_{23}(x), \quad l_{1}+l_{2}<x \leq l_{1}+2 l_{2}, \\
y_{34}(x), \quad l_{1}+2 l_{2}<x \leq 2\left(l_{1}+l_{2}\right) .
\end{array}\right.
$$

The stress state of the system is defined by the values of the bending moment, the shearing force, the normal and the shear stresses:

$M(x)=E J \frac{d^{2}}{d x^{2}} y(x), Q(x)=E J \frac{d^{3}}{d x^{3}} y(x)$,

$\sigma(x)=M(x) / W, \tau(x)=Q(x) S_{\max } / b J$,

where $S_{\max }=b h^{2} / 8, J=b h^{3} / 12, W=b h^{2} / 6$ are the geometrical characteristics of the flat spring's cross section; $b$ and $h$ are the width and the thickness of the flat spring, correspondingly.

If the nodal loads vector is presented as the concentrated force with the amplitude value $F$, which acts on the local oscillatory mass in the central cross-section, thus, by solving equations (18), (20)-(22), in turn, we obtain the corresponding diagrams of deflection, rotation angle, shearing forces and bending moments when the rod bends. Calculation of the maximum equivalent stresses in the corresponding cross-sections of the elastic element and for the corresponding design diagrams is very important for the proposed variant of implementation of the asymmetric stiffness characteristic.

The values of the maximal bending stresses are defined using the well-known formulas (the indexes denote the stresses for corresponding design diagrams):

$\sigma_{\max I}=\frac{M_{\max I}}{W}, \sigma_{\max I I}=\frac{M_{\max I I}}{W}$,

where $M_{\max I}=\frac{F L}{8}, \quad M_{\max I I}=\left[\bar{Z}_{12}\right]_{4}=\frac{F l_{2}\left(l_{1}+2 l_{2}\right)}{2\left(l_{1}+4 l_{2}\right)}$ are the maximal bending moments in the dangerous cross-sections of the rod systems discussed.

The maximal shearing stress in the central cross-sections of the rod can be defined as:

$\tau_{\max I}=\tau_{\max I I}=\frac{1.5 F}{b h}$,
The equivalent von Mises bending stress is as follows:

$\sigma_{e q}=\sqrt{\sigma_{\text {max }}^{2}+4 \tau_{\text {max }}^{2}}$.

The stress-strain state analysis of the rod systems can be carried out by the value of the displacement $y_{m}$ of the local mass or by the value of the restoring force which is defined by the given displacement for the corresponding diagrams using the appropriate formulas: $F_{I}=c_{I} y_{m}, F_{I I}=c_{I I} y_{m}$. Considering that $y_{m}=$ $0.24 \mathrm{~mm}$, we obtain the diagram of the form I with $\sigma_{e q I}=$ $23.2 \mathrm{MPa}$; for the diagram of the form II we obtain $\sigma_{e q I I}=$ 59.5 MPa. The diagram of the deflection and equivalent stresses for a half of the elastic element (considering symmetry of the rod system) is presented in Fig. 6 .

a)

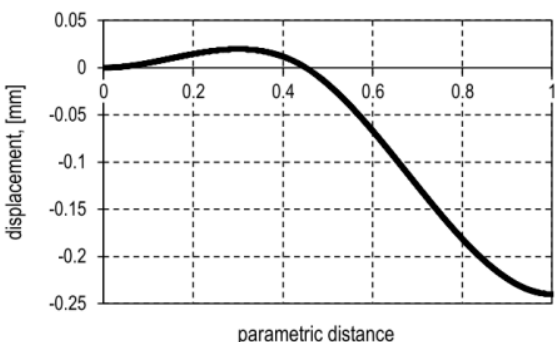

b)

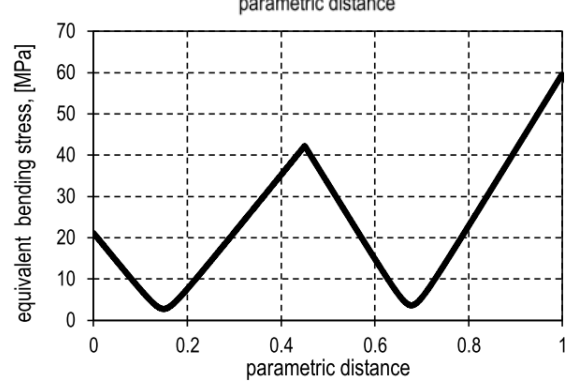

Fig. 6. Graphical dependencies of deflection (a) and equivalent bending stress (b) for the half-rod

Considering the reactions in the intermediate supports (caused by the shearing force), the check on the contact stresses between the cylindrical support and the flat spring is carried out based on Hertzian contact stress theory. The contact pressure is defined by the corresponding formula (Shigley, 2011):

$p_{\max }=\frac{2 Q_{1}}{\pi b t}$

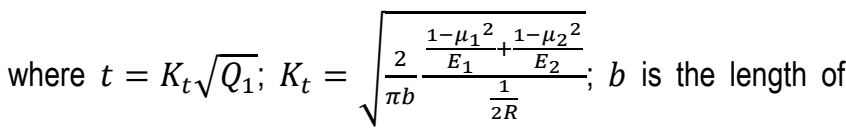
the contact area (i.e., the width of the flat spring); $R$ is the radius of the intermediate support; $E_{1}, E_{2}$ and $\mu_{1}, \mu_{2}$ are the elasticity modula and the Poisson's ratio of the materials being in the contact; $Q_{1}$ is the support action on a spring, which can be determined at the sum of reactions (shearing forces) in the rod's node:

$$
Q_{1}=\left|\left[\overline{\boldsymbol{Z}}_{\mathbf{0 1}}\right]_{3}+\left[\overline{\mathbf{Z}}_{\mathbf{1 2}}\right]_{1}\right|=\frac{F_{I I}\left(l_{1}{ }^{2}+4 l_{1} l_{2}+3 l_{2}{ }^{2}\right)}{2 l_{1}\left(l_{1}+4 l_{2}\right)} .
$$

The components of the spatial stressed state vector are determined by the following formulas (Shigley, 2011): 
$\sigma_{x}=-p_{\max }\left(\frac{1+2 \zeta_{b}^{2}}{\sqrt{1+\zeta_{b}^{2}}}-2\left|\zeta_{b}\right|\right)$

$\sigma_{y}=-p_{\max } \frac{1}{\sqrt{1+\zeta_{b}^{2}}}$

$\sigma_{z}=-2 \mu_{1} p_{\max }\left(\sqrt{1+\zeta_{b}^{2}}-\left|\zeta_{b}\right|\right)$.

where $\zeta_{b}=y / t=0.786$ is used for the calculations considering the maximal stress values.

Herewith, the main stresses are equal to the following axial components:

$\sigma_{1} \equiv \sigma_{x}, \sigma_{2} \equiv \sigma_{z}, \sigma_{3} \equiv \sigma_{y}$.

The maximal shear stress and the equivalent stress in the contact zone are as follows:

$\tau_{\max }=\frac{\sigma_{1}-\sigma_{3}}{2}$,

$\sigma_{\max }{ }^{\text {(contact) }}=$

$\sqrt{\frac{1}{2}\left[\left(\sigma_{1}-\sigma_{2}\right)^{2}+\left(\sigma_{2}-\sigma_{3}\right)^{2}+\left(\sigma_{3}-\sigma_{1}\right)^{2}\right]}$.

The compliance of the intermediate supports depends on the relative displacement $\Delta_{c}$ of the flat spring and the support in contact zone can be determined by the following equation (Pisarenko et al., 1988):

$\Delta_{\mathrm{c}}=0.83 \sqrt[3]{\frac{Q_{1}^{2}}{R}\left(\frac{1-\mu_{1}^{2}}{E_{1}}+\frac{1-\mu_{2}^{2}}{E_{2}}\right)^{2}}$.

The stiffness coefficient in the contact zone is $c_{y}=Q_{1} / \Delta_{c}$. For steel-steel contact problem the formulas are as follows:

$\Delta_{\mathrm{C}}=1.23 \sqrt[3]{\left(\frac{Q_{1}}{E}\right)^{2} \frac{1}{R}}$

$c_{y}=0.81 \sqrt[3]{Q_{1} R E^{2}}$.

The last formula allows taking into account contact stiffness of the intermediate supports during implementation of the vibroimpact operation modes. In particular, assuming that its value is parametrically dependent on the contact force, we obtain the dependence of contact stiffness on the instantaneous displacement $y(t)$ of the local mass.

\section{DYNAMIC ANALYSIS}

In order to perform the dynamic analysis (Dyachenko et al., 2017), there is studied a model of forced oscillations taking into account instantaneous change of the frequency parameters of the rod system due to switching the stiffness characteristics and considering the contact stiffness of the intermediate supports:

$$
\begin{aligned}
y^{\prime \prime}(t)+2 n \cdot y^{\prime}(t) & +\left\{\begin{array}{c}
\omega_{0 I}^{2} \cdot y(t), y(t) \geq 0 \\
\Omega_{0 I I}[y(t)]^{2} \cdot y(t), y(t)<0
\end{array}\right. \\
& =f \cdot \sin (\omega t),
\end{aligned}
$$

where: $\quad n=b / 2 m, \quad b=2 m \omega \zeta, \quad \zeta=0.15, \quad f=F / m$, $F=600 \mathrm{~N}$.
The kinematic characteristics (Fig. 7) of the local oscillating mass motion are obtained using the numerical method RADAU while solving (35). The characteristics have asymmetric nature confirming the presence of the vibro-impact mode.

a)

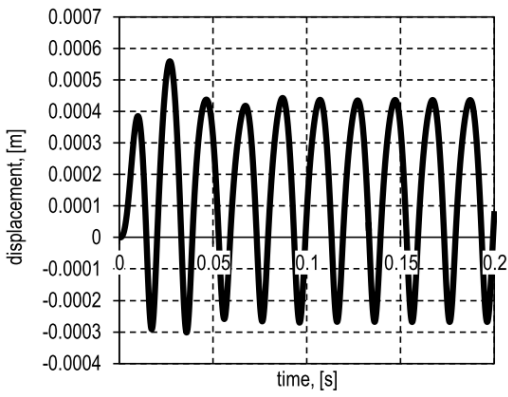

b)

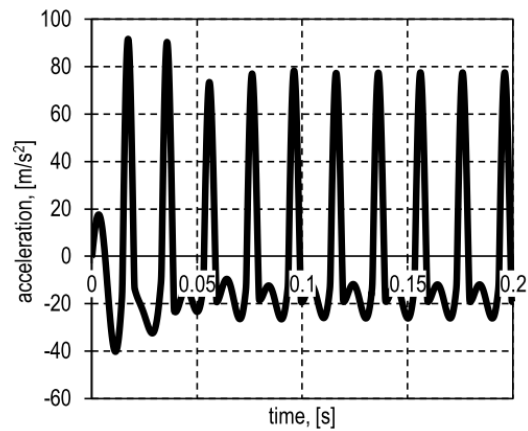

Fig. 7. Time dependencies of the dynamic displacement (a) and the acceleration $(b)$ of the local mass

The obtained system has the following amplitude-frequency characteristic (Fig. 8), the resonance peak of which is determined by the following value of the natural frequencies of free oscillations (Yevstignejev, 2008):

$\Omega_{0}=\frac{2 \omega_{0 I} \omega_{0 I I}}{\omega_{0 I}+\omega_{0 I I}}=418.67 \frac{\mathrm{rad}}{\mathrm{s}}$.

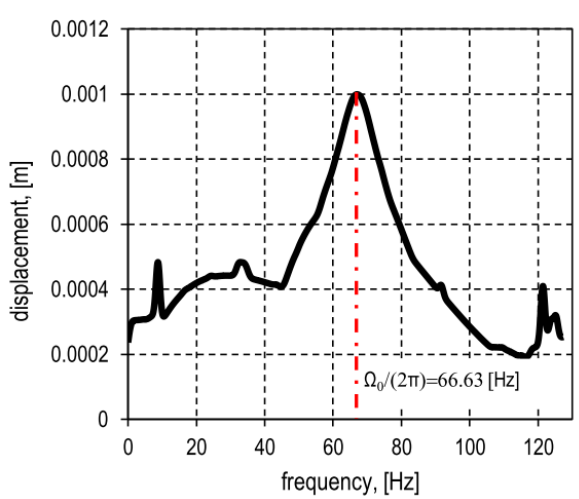

Fig. 8. Amplitude-frequency characteristic of the system

Multiple subharmonic and superharmonic resonances, which are analyzed in publications of Belovodskiy (2015), Luo (2007, 2009) and Simon (2014), can be observed on the obtained amplitude-frequency characteristic (Fig. 8).

Considering that in Eg. (27) the contact force depends on the instantaneous displacement $y(t)$ of the local mass, the parametric dependency of the contact stiffness on the contact force is constructed in Fig. 9 and has the explicitly nonlinear character. 


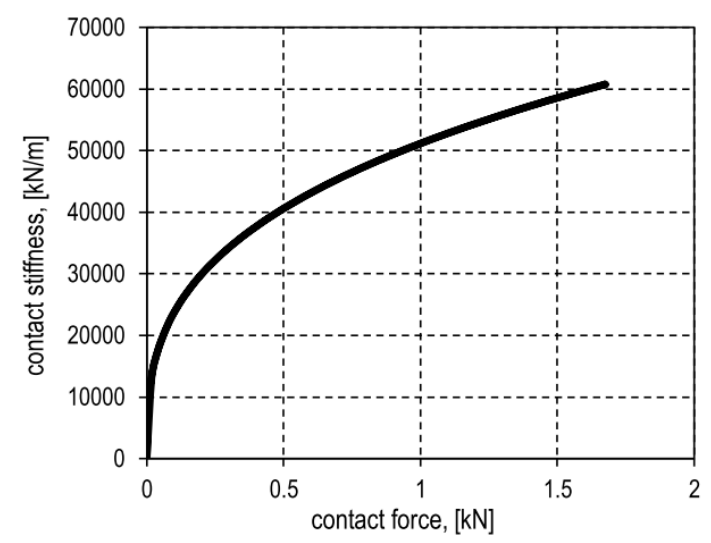

Fig. 9. Dependency of the stiffness coefficient of the intermediate support on the contact force (on the local mass displacement)

Herewith, the equivalent bending stress and the contact force are determined by the working conditions of the rod system:

$\sigma_{e q}(t)=\left\{\begin{array}{l}\sigma_{e q I}(t), y(t) \geq 0, \\ \sigma_{e q I I}(t), y(t)<0\end{array}\right.$.

$Q_{\mathrm{c}}(t)=\left\{\begin{array}{c}0, y(t) \geq 0, \\ \frac{F_{I I}[y(t)]\left(l_{1}{ }^{2}+4 l_{1} l_{2}+3 l_{2}{ }^{2}\right)}{2 l_{1}\left(l_{1}+4 l_{2}\right)}, y(t)<0,\end{array}\right.$

where $F_{I I}[y(t)]=c_{I I}[y(t)] y(t)=m \Omega[y(t)]^{2} y(t)$ is the restoring force in the central cross-section of the rod system.

The instant dependencies of the equivalent bending stresses and the contact stresses are presented in Fig. 10 and have the asymmetric impulsive character. The obtained results shows that the maximum values of the bending and contact stresses take place during the contact of the flat spring with the cylindrical supports.

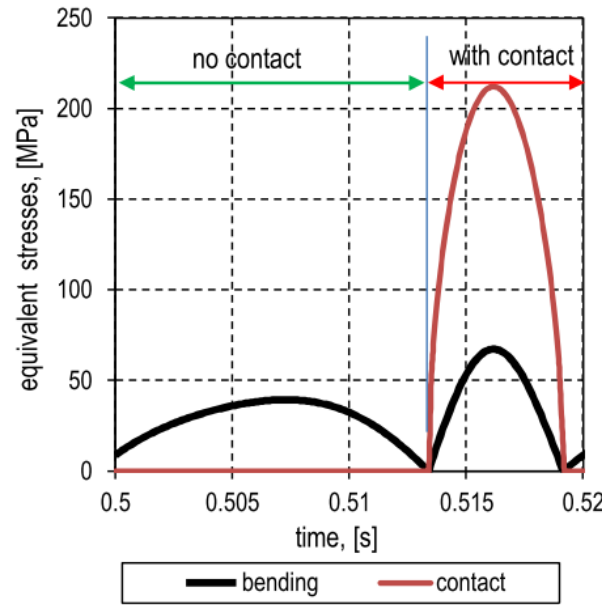

Fig. 10. Time dependency of the equivalent bending stresses and the contact stresses of the vibro-impact rod system

The bending stress is changing linearly and the contact stress is nonlinear in relation to the displacement of the local mass (Fig. 11 , a). As an additional result, let us present the parametric dependency of the calculated stress values (Fig. 11a) and the restoring force of the flat spring (Fig. 11b) in respect to the instantaneous displacement of the local mass. a)

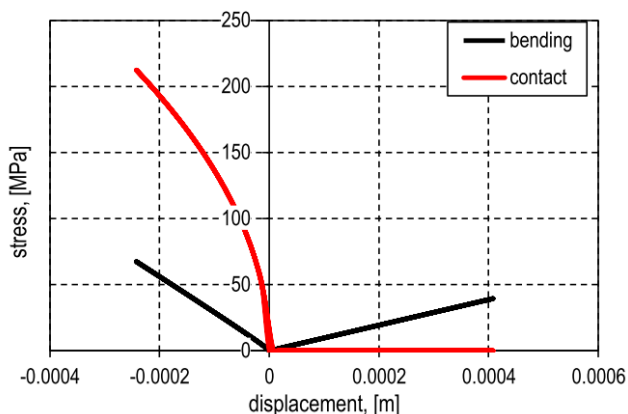

b)

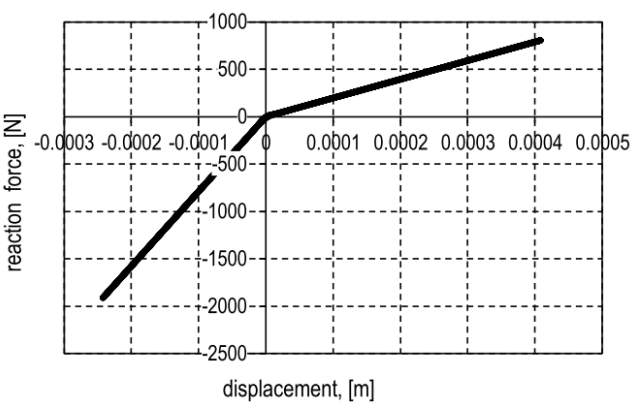

Fig. 11. Parametric dependency of the calculated stress values (a) and the restoring force (b) of the spring in respect to the displacement of the local mass

The synthesized asymmetric stiffness characteristic taking into account compliance of the intermediate supports (contact zone pliability) can be presented as:

$R[y(t)]=\left\{\begin{array}{l}c_{I} y(t), y(t) \geq 0 \\ c_{I I} y(t), y(t)<0\end{array}\right.$.

Subsequently, the considered elastic element can be calculated by well-known techniques in order to ensure necessary durability and endurance taking into account bending and contact stiffness and stress asymmetry (Kogaev, 1977). The similar results are presented in the publication (Gursky and Kuzio, 2016). Complex accounting of the bending and contact stresses is carried out using the reduced coefficient of strength, which should not exceed the permissible value.

\section{CONCLUSIONS}

A complex dynamic analysis of the vibro-impact system based on the rod system is carried out using the finite element method. Compliance of the intermediate supports is considered and the analytical equation for calculating the natural frequency of free oscillations of such system is derived. The calculations of the contact stiffness are presented and modeling of oscillations of the vibro-impact system is performed taking into account stiffness of the intermediate supports. On the basis of the results of the dynamic analysis the stresses checking is carried out considering the bending conditions and the contact stiffness. The expediency of this approach is substantiated by the obtained formulas which are used while carrying out design calculations and allow to perform the efficiency evaluation of the vibro-impact modes implementation meeting the requirement imposed on strength of the basic system's element. 


\section{REFERENCES}

1. Babitsky V. (2013), Theory of vibro-impact systems and applications, Springer Science \& Business Media.

2. Bednarski Ł., Michalczyk J. (2017), Modelling of the working process of vibratory conveyors applied in the metallurgical industry, Archives of Metallurgy and Materials, 62(2), 721-728.

3. Belovodskiy V.N., Bukin S.L., Sukhorukov M.Y., Babakina A.A. (2015), 2:1 superharmonic resonance in two-masses vibrating machine, Journal of Vibration Engineering \& Technologies, 3(2), 123-135.

4. Clough Ray W., Joseph Penzien. (1995), Dynamics of Structures, Berkeley: Computers \& Structures.

5. David V. Hutton. (2004), Fundamentals of finite element analysis, Editorial McGraw - Hill, USA.

6. Despotović Ž.V., Lečić M., Jović M. R., Durić A. (2014), Vibration control of resonant vibratory feeders with electromagnetic excitation, FME Transactions, 42(4), 281-289.

7. Dyachenko P., Chychuzhko M., Al-Ammouri A. (2017). Development and application of computer model to study the modes of dynamic loading in mechanical oscillatory systems. EasternEuropean Journal of Enterprise Technologies, 1(85), 42-49.

8. Filimonikhin G., Yatsun V. (2017). Conditions of replacing a singlefrequency vibro-exciter with a dual-frequency one in the form of passive auto-balancer. Naukovyi Visnyk Natsionalnoho Hirnychoho Universytetu, 1, 61-68.

9. Gursky V., Kuzio I. (2016), Strength and durability analysis of a flat spring at vibro-impact loadings, Eastern-European Journal of Enterprise Technologies, 5, 7(83), 4-10.

10. Kogaev V.P. (1977), Raschetu na prochnost pry napriazhenyiakh, peremennukh vo vremeny [Calculation of strength under stresses variable in time], Mashinostroenie, Moscow, (in Russian).

11. Luo G., Ma L., Lv X. (2009), Dynamic analysis and suppressing chaotic impacts of a two-degree-of-freedom oscillator with a clearance, Nonlinear Analysis: Real World Applications, 10(2), $756-778$
12. Luo G., Zhang Y., Xie J., Zhang J. (2007), Vibro-impact dynamics near a strong resonance point, Acta Mechanica Sinica, 23(3), 329-341.

13. Nadutyi V.P., Sukharyov V.V., Belyushyn D.V. (2013), Determination of stress condition of vibrating feeder for ore drawing from the block under impact loads, Metallurgical \& Mining Industry, 5(1), 24-26.

14. Pavel V. Krot. (2010), Dynamics and diagnostics of the rolling mills drivelines with non-smooth stiffness characteristics, Proceedings of the 3rd International Conference on Nonlinear Dynamics, Kharkov, Ukraine, 115-120.

15. Pisarenko G.S., Yakovlev A.P., Matveev V.V. (1988), Spravochnyk po soprotyvlenyyu materyalov [Handbook on strength of materials, Naukova Dumka, Kiev, (in Russian).

16. Shigley Joseph Edward. (2011), Shigley's mechanical engineering design, Tata McGraw-Hill Education.

17. Simon P., Reuss P, Gaul L. (2014), Identification of sub- and higher harmonic vibrations in vibro-impact systems, Nonlinear Dynamics, 2, 131-140.

18. Sokolov I.J., Babitsky V.I., Halliwell N.A. (2007), Autoresonant vibro-impact system with electromagnetic excitation, Journal of Sound and Vibration, 308, 375-391.

19. Vladislav Yevstignejev (2008), Application of the complete bifurcation groups method for analysis of strongly nonlinear oscillators and vbro-impact systems, Riga, Summary.

20. Yoon J. Y., Kim B. (2015), Vibro-impact energy analysis of a geared system with piecewise-type nonlinearities using various parameter values, Energies, 8(8), 8924-8944. 